\title{
Study on the Evaluation and Operation Efficiency of Elevator Service Quality Based on MATLAB
}

\author{
Sheng-Li FAN ${ }^{1, a,{ }^{*}}$ \\ ${ }^{1}$ College of Management, Northwest University for Nationalities, Lanzhou, China \\ afan120120@sina.com \\ ${ }^{*}$ Corresponding author
}

Keywords: MATLAB, Statistical characteristics, Service quality, Operational efficiency.

\begin{abstract}
A bstract. The statistical characteristic of lift transport system is the statistics laws which study the lift traffic with the statistical methods. The rate of 5-minute load (CE), the number of lift(N), the average aperture time(AI), the time of average travel(AP) and the acceleration distance(Sa) are the parameters of describing the statistical characteristics. This thesis will be mainly based on the statistical characteristics of the lift transport system, using statistical methods to design a specific and simulated model analyzing the lift service quality and operational efficiency for medium and large office building. It aims to make the users according to the simulation result analyze the design parameters reasonably, and lead to practice.
\end{abstract}

\section{Introduction}

Elevator traffic configuration theory and engineering application are always promote each other and common development. The statistical characteristic of elevator traffic system refers to the statistical regularity of elevator traffic statistical method. For example, as output components: 5 minute rates $\mathrm{CE}$, the number of elevator $\mathrm{N}$, the average number of elevator clearance time $\mathrm{AI}$, average travel time $\mathrm{AP}$ and the acceleration distance $\mathrm{Sa}$ are the parameters to describe the statistical characteristics.

\section{A nalysis of E levator Service Q uality and O perating E fficiency Principle}

\section{General Description}

The elevator traffic system is a multi input and multi output system overall. The main external factors influence the efficacy group service quality and operation of the elevator has characteristics of buildings, elevator equipment characteristics and the elevator operating characteristics.

\section{Describe of Input and its R elationship with Intermediate Variables}

\section{Type of Building}

The type of building is $\mathrm{m} 1$. The type of building is usually divided into 5 kinds: office buildings, residential buildings, hotels, hospitals and department store. Building types can expect to such as determine the rated speed Ve and rated load variables Re, and so on.

\section{Building Scale}

Building scale is $\mathrm{m} 2$. The size of the building includes the building of every layer of the construction area of $\mathrm{Ai}$, each layer of the effective utilization coefficient ${ }_{\mid} \hat{\mathrm{A}}$, elevator service layers fm. The size of the building can directly determine the elevator using cardinal Q, car travel distance SL and short interval class service level $n$.

\section{E levator Dragging C ategory}

Elevator dragging category is $\mathrm{m} 3$.

Elevator equipment types, are generally divided into four types:

(1) the AC single speed gear 
(2) dc gear

(3) AC double speed gear

(4) brushless DC gear

Through the elevator dragging category $\mathrm{m} 3$ can directly determine the short interval single station running time of TR, the acceleration distance $\mathrm{Sa}$ and the acceleration time ta. For example, by the elevator dragging category $\mathrm{m} 3$, rated speed Ve and short interval average running distance $\mathrm{S}$ can determine the shortest interval single station running time of tr.

The elevator dragging category $\mathrm{m} 3$ can directly determine the short interval single station running time of TR, the acceleration distance $\mathrm{Sa}$ and the acceleration time ta.

$\mathrm{m} 3$ and Ve can be determined by Sa and ta. For example, the tooth direct-current ladder, when $\mathrm{Ve}=1.75 \mathrm{~m} / \mathrm{s}$, the maximum acceleration $\mathrm{a}=1.1 \mathrm{~m} / \mathrm{s}$, variable acceleration time is $\mathrm{t}=0.75 \mathrm{~s}$, the corresponding acceleration time and the acceleration distance are $t a=2.35 \mathrm{~s}$ and $\mathrm{S}=2.04 \mathrm{~m}$.

$\mathrm{m} 3$ and Ve can be determined by Sa and ta.

\section{E levator Service}

The elevator service mode indicated by $\mathrm{m} 4$. The operation mode of the elevator. Can be divided into 6 types:

(1) single speed

(2) and the interval speed

(3) single interval speed

(4) a single high-level service

(5) the layer or interlayer service

(6) a single underlying service

\section{E levator C ar Door C ategory}

The elevator door category $\mathbf{J}$. In the lift formula $\mathrm{J}$ represents the car door width. All the physical quantities here $\mathbf{J}$ refers to the cabin door, including door way. The elevator door is not directly determine the door unit time TD and each passenger access time tp. For example, the automatic door AC Lift double doors, door entrance width $900 \mathrm{~mm}$. To open the door, electric door opening time of $\mathrm{t}=3.2 \mathrm{~s}$.

$\mathrm{m} 1, \mathrm{~m} 2, \mathrm{~m} 3, \mathrm{~m} 4$ and $\mathrm{j}$ can both directly (or expected) to determine the elevator traffic system of one or several physical quantities, so is the system input component of the whole.

\section{Describtion of Output and its R elationship with Intermediate Variables}

\section{Operation Period of Elevator}

Elevator operation period of RTT refers to the elevator running a week time required, namely the car from the base station, the passengers to each floor, back to the base station need time.

The entry and exit of passengers total time: $\mathrm{Tp}={ }_{i}$ \#(t1i+t5i)

Switch door total time: $\mathrm{Td}=i \nexists(\mathrm{t} 2 \mathrm{i}+\mathrm{t} 4 \mathrm{i})$

Running total time: $\operatorname{Tr}=i$ A $3 \mathrm{i}$

Then

Operation cycle: $\mathrm{RTT}=\mathrm{Tp}+\mathrm{Td}+\mathrm{Tr}$

For the single express service, the formula can also be expressed as:

When $\mathrm{S}<2 \mathrm{Sa}$ : 


$$
R T T=t_{a}+1.1 t_{d}+0.88 R e t p+\frac{h(f m-1)}{V e}+n(t r+1.1 t d)-\left(\frac{n-1}{n}\right)^{0.8 R e}
$$

When $\mathrm{S}>2 \mathrm{Sa}$ :

$$
\mathrm{RTT}=(\operatorname{ta}+1.1 \mathrm{td})\left[1+\mathrm{n}-\mathrm{n}\left(\frac{\mathrm{n}-1}{\mathrm{n}}\right)^{0.8 \mathrm{Re}}\right]+\frac{2 \mathrm{~h}(\mathrm{fm}-1)}{\mathrm{Ve}}+0.88 \mathrm{Retp}
$$

\section{Average Travel Time}

The average travel time of AP refers to the elevator car door operating from the departure of arriving at the destination by time statistic average value, the one-way speed service mode, the calculation methods such as:

$$
\mathrm{Ap}=\mathrm{RTT}-(\mathrm{ru}+2)-1.1 \mathrm{td}
$$

\section{Average Clearance Time}

The average time between AI refers to a group of $\mathrm{N}$ elevator, every two adjacent station to station time difference. Expressed as:

$$
\mathrm{AI}=\frac{\mathrm{RTT}}{\mathrm{N}}
$$

\section{A verage Waiting Time}

The average time of AWT refers to the average passenger waiting time waiting for the elevator, expressed as:

$$
\mathrm{AWT}=0.6 \mathrm{AI}
$$

\section{M inutes 0 ccupancy R ate}

5 minutes carrying rate of $\mathrm{CE}$ is 5 minutes and the total number of elevator conveyer elevator technology use ratio, is an important indicator to set the vertical transport efficiency of the elevator buildings. Expressed as:

$$
\mathrm{CE}=\frac{5 \times 60 \mathrm{rN}}{\mathrm{RTT}}
$$

\section{The Simulation Model}

\section{Simplified System M odel Principle}

(1) the experience data adopt national standard;

(2) simulation system is designed only for one building -- large and medium-sized office building concrete.

\section{Determine the O utput}

(1) the elevator operation cycle RTT (n)

(2) the average travel time of Ap (n)

(3) the average clearance time AI (n)

(4) the average waiting time of AWT (n)

(5) 5 minutes carrying rate of CE (n) 


\section{Final Input Variables}

(1) Highest number of elevator service fm

(2) Total number of $\mathrm{N}$

(3) Average height of each floor $h$

\section{Simulation System}

\section{Input the fm, h, N, to simulation.Simulation R esults and Analysis}

\section{Simulation R esults}

Five occupancy rate $\mathrm{CE}$ and service layers $\mathrm{n}$ relationships are shown in figure:

Relationship between elevator operation period of RTT and the average travel time of AP and the service layer of the $n$ is shown in Figure

The average time between AI and the average waiting time of AWT and service layers $n$ relationships are shown in Figure

For the most high $(\mathrm{n}=18)$ the simulation data for:

Elevator operation cycle RTT $=259.5634(\mathrm{~s})$

The average travel time of $\mathrm{AP}=73.1234(\mathrm{~s})$

The average time between $\mathrm{AI}=14.4202(\mathrm{~s})$

The average waiting time of AWT $=8.65211$ (s)

5 minutes carrying rate of $\mathrm{CE}=2.9 \%$

\section{Simulation Results}

Based on the above image shows, the income of this simulation system simulation results accord with the reality of the basic statistical law. The following will analyze:

According to the calculation, the highest level in the $(n=18)$ when the output of the expected value respectively:

Elevator operation cycle RTTe $=235$ (s)

The average travel time of $\mathrm{APe}=60(\mathrm{~s})$

The average time between $\mathrm{AIe}=16(\mathrm{~s})$

The average waiting time of $\mathrm{AWTe}=13(\mathrm{~s})$

5 minutes carrying rate of $\mathrm{CEe}=1.4 \%$

By the comparison of the above data, most of the parameters meet the design requirements, but the CEe error is relatively large, further calculation, the actual number of passengers five minutes for 74 people, 36 people far more than expected, suggesting that the elevator is free in most cases, this configuration is not reasonable.

To change the setting the number of elevators $\mathrm{N}$ value, using this simulation system, we find that, under normal circumstances, only need 2 elevators will be able to meet the requirements, the operation efficiency of the elevator is relatively high. But considering the elevator is in the middle of the day is very uneven, in peak flow conditions, two elevators can not meet the requirements.

\section{Summary}

Based on the principle of elevator configuration optimization of transportation system, defines the effectiveness of the quality of service and operation evaluation standard statistical parameters. According to the practical requirements, set up a special for the twenty-five layer below the office building of the Matlab simulation system by using the method of statistics, and then through the simulation of actual examples, to prove the feasibility of this simulation system.

The simulation system, have done the relevant set of building type, the floor height and number of floors, and through the query graph, table and the formula to calculate the statistical parameters to obtain the desired value, then the simulation using the simulation system, the statistical characteristics parameter values, were compared with the expected value. To evaluate the rationality of design 
configuration and the optimum design in order to achieve efficiency improving service quality and operation of the elevator.

\section{References}

[1] Zhu Dewen. "Elevator traffic system of intelligent control and application", Jilin University press, 2002, pp20-72.

[2] Li Xianhong. "MATLAB 7.x interface design and compilation techniques", electronics industry press,, 2006.

[3] Li Zhenshan, Shao Cheng. "Control and decision of present situation and development trend of elevator group control technology", 2005, 20 (12), pp1321-1331.

[4] Li Zhenshan, Shao Cheng. "based on bi level programming model of elevator traffic system group optimization", Journal of Shenyang Construction University, 2007 Vol.23, pp170-176.

[5] Yang Zhenshan. "the elevator vertical transportation system configuration and optimization scheduling problem research", Ph.D. thesis, Dalian University of Technology, Dalian, 2008. 